\title{
Frequency Tuning of Work Modes in Z-Axis Dual-Mass Silicon Microgyroscope
}

\author{
Lu Xu, ${ }^{1,2}$ Hongsheng Li, ${ }^{1,2}$ Yunfang Ni, ${ }^{1,2}$ Jia Liu, ${ }^{1,2}$ and Libin Huang \\ ${ }^{1}$ School of Instrument Science and Engineering, Southeast University, Nanjing 210096, China \\ ${ }^{2}$ Key Laboratory of Micro-Inertial Instrument and Advanced Navigation Technology of Ministry of Education, \\ Southeast University, Nanjing 210096, China \\ Correspondence should be addressed to Hongsheng Li; hsli@seu.edu.cn
}

Received 29 April 2014; Revised 21 August 2014; Accepted 30 November 2014; Published 15 December 2014

Academic Editor: Laurent Francis

Copyright (C) $2014 \mathrm{Lu} \mathrm{Xu}$ et al. This is an open access article distributed under the Creative Commons Attribution License, which permits unrestricted use, distribution, and reproduction in any medium, provided the original work is properly cited.

\begin{abstract}
Frequency tuning of work modes in the silicon vibratory gyroscope is studied by the theoretical, numerical, and experimental methods in this paper. First, the schematic structure and simplified kinematics model of the gyroscope were presented for deducing the natural frequencies. Then, the width and length of support beams were optimized to tune work frequencies at their designed value. Besides, the frequency difference was experimentally tested and manually tuned by varying the voltage applied on the tuning capacitors. The test on a prototype showed that the difference could be localized between $-55.8 \mathrm{~Hz}$ and $160.2 \mathrm{~Hz}$ when the tuning voltage limit is $20 \mathrm{~V}$. Finally, a frequency control loop was developed to automatically tune the sense frequency toward the drive frequency. Both the theoretical analysis and numeric simulation show that the difference is stabilized at $0.8 \mathrm{~Hz}$ when no Coriolis force or quadrature coupling force is applied. It is proved that the frequency difference is successfully tuned by modifying the size of support beams before fabrication as well as the voltage applied on the tuning capacitors after fabrication. The automatic tuning loop, used to match the work modes, is beneficial to enhance the performance of the gyroscope as well as its resistance to environment disturbances.
\end{abstract}

\section{Introduction}

Silicon microgyroscopes have a lot of merits, such as low power consumption, small volume and weight, high shock resistivity, and suitability for batch fabrication, but their performance is lower than conventional mechanical, rotating wheel, fiber-optical, and ring laser gyroscopes $[1,2]$. In order to broaden silicon microgyroscopes' application, a lot of work has been done on improving their performance, such as modification and optimization of the gyroscope structure, the fabrication process, the quality of vacuum packaging, and the detect circuit as well as the control system. Among these methods, the research on improvement of the structure and the circuit and system is the most economical.

The dual-mass gyroscopes are now popular for their effective rejection of common-mode disturbance, so many institutions [3-7] have done much research on them. It is known that the natural frequencies of work modes are key factors for performance improvement in silicon gyroscopes such as the bandwidth and the sensitivity $[1,8]$. However, due to the fabrication imperfection, it is difficult to control the work frequencies at their designed value, causing the performance to be unpredictable. Therefore, some methods for postfabrication modification of the work frequencies were studied, such as the selective deposition of polysilicon [9], the localized thermal stressing effect [10], and the negative electrostatic stiffness effect $[7,8,11-13]$. From the three methods, the electrostatic tuning method is popular for low cost, little damage to the gyroscope structure, and easy adaptability in different gyroscopes.

The electrostatic tuning method can be realized by controlling the tuning voltage manually or automatically. The automatic control can be realized based on the frequency response characteristic of the mass-spring resonator. When the resonator vibrates at the natural frequency, the vibration amplitude achieves its maximum value and the phase delay is 90 degree. Thus, [12] designed the automatic control loop using the phase characteristic but the controlled frequency difference is influenced by the quadrature coupling. The method by seeking the maximum amplitude is studied by 


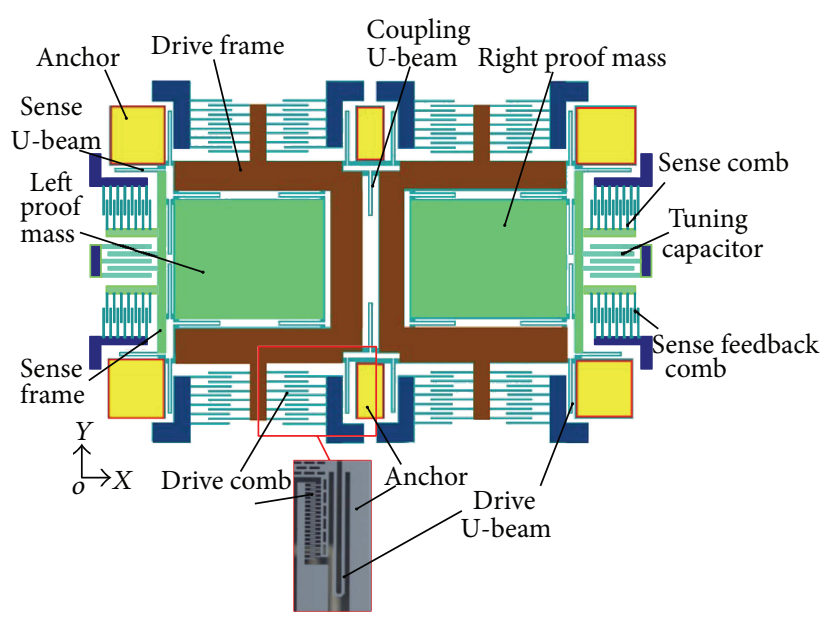

(a) Schematic structure of the gyroscope

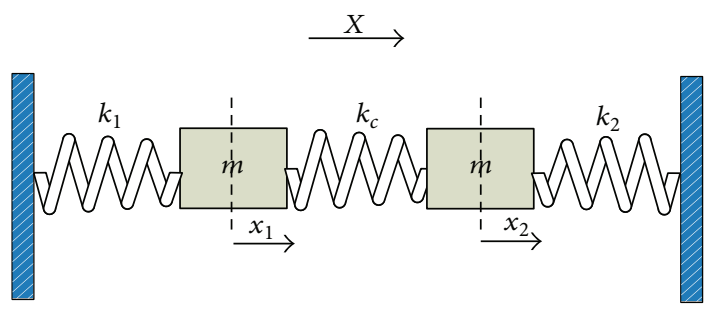

(b) Simplified kinematics model for the dual-mass vibration in the gyroscope

FIGURE 1: Structure of the dual-mass silicon microgyroscope.

[13], but when the input angular rate is not constant, the controlled frequency is not stable. Theoretically and numerically, the frequency control algorithm presented in this paper is less influenced by the quadrature coupling and the varied input angular rate, especially when they are small.

In this paper, the methods for frequency localization of the work modes are detailed from two aspects: structure optimization and the postfabrication control. In Section 2, based on the structure of a dual-mass silicon microgyroscope, the simplified kinematics model is firstly built to deduce the natural frequencies for the in-phase and antiphase vibrations. Then, in Section 3, a finite element model of the gyroscope is created using Ansys to analyze the relationship between the work frequencies and the size of support beams. Besides, the capacitors of parallel plates are designed in sense frames for frequency tuning by means of the negative electrostatic stiffness effect. The frequency sweeping experiments on a fabricated gyroscope prototype prove that frequency tuning of the work modes is achieved by adjusting the size of support beams as well as by manually controlling the voltage applied on the tuning capacitors. Finally, in Section 4, the automatic frequency tuning of work modes is realized by a closed frequency-control loop.

\section{Structure}

As shown in Figure 1(a), the gyroscope is comprised of two symmetric single-mass gyroscopes coupled with some links, which are coupling U-beams here [6]. Each single-mass gyroscope contains a drive frame, a proof mass, and a sense frame, serving as the energy source, the energy transferring unit, and the output unit, respectively. The specific arrangement of multiple frames makes the sense frames move only in the sense direction ( $Y$-axis), maximally reducing the disturbance from drive frames, such as the quadrature force $[14,15]$.

When subject to a constant out-of-plane input angular rate $\Omega_{\text {in }}$, the stable vibration amplitude of sense frames is approximated as shown in

$$
A_{y}=\frac{2 A_{d} \omega_{d} \Omega_{\mathrm{in}}}{\sqrt{\left(\omega_{y}^{2}-\omega_{d}^{2}\right)^{2}+\left(\omega_{y} \omega_{d} / Q_{y}\right)^{2}}},
$$

where $A_{d}$ is the vibration amplitude of drive frames, $Q_{y}$ is the quality factor of sense mode, and $\omega_{d / y}$ is the natural frequency of drive/sense mode. It is easily found that the mechanical sensitivity $S=A_{y} / \Omega_{\text {in }}$ is larger when the frequency difference between $\omega_{y}$ and $\omega_{d}$ is decreased. However, the mechanical working bandwidth BW $=0.54\left|\omega_{y}-\omega_{d}\right|$ decreases. Thus, the size of support beams is optimized in the structure design course and the frequency tuning electrodes are designed in the sense frames, so as to obtain an optimal frequency difference between work modes as well as large sense amplitude.

In Figure 1(b), the simplified kinematics model [16], neglecting the damping effect, is developed for analyzing the motion of the dual-mass gyroscope in the drive or sense direction. $k_{1}$ and $k_{2}$ are the effective system stiffness between 
each vibrating mass and the substrate, $k_{c}$ is the coupling stiffness between the two vibrating masses, and $m$ is the effective vibrating mass. The kinematics analysis for the dualmass system is displayed in [17]

$$
\begin{gathered}
-k_{2} x_{2}-k_{c}\left(x_{2}-x_{1}\right)=m \ddot{x}_{2}, \\
k_{c}\left(x_{2}-x_{1}\right)-k_{1} x_{1}=m \ddot{x}_{1},
\end{gathered}
$$

from which the natural frequencies are derived as shown in the following:

$$
\begin{aligned}
& f_{\text {ip }}=\frac{1}{2 \pi} \sqrt{\frac{k_{1}+k_{2}+2 k_{c}-\sqrt{\left(k_{1}-k_{2}\right)^{2}+4 k_{c}^{2}}}{2 m}}, \\
& f_{\text {op }}=\frac{1}{2 \pi} \sqrt{\frac{k_{1}+k_{2}+2 k_{c}+\sqrt{\left(k_{1}-k_{2}\right)^{2}+4 k_{c}^{2}}}{2 m}} .
\end{aligned}
$$

The frequency in (3) corresponds to the in-phase vibration, namely, the two masses translating in the same direction, which is considered as a disturbing mode for the dual-mass gyroscope function. Similarly, the frequency in (4) is for the antiphase vibration. In the gyroscope, the work modes are the opposite vibrations in the drive and sense direction, so the work frequencies can be obtained from (4).

\section{Frequency Localization}

3.1. Tuning Effect of Support Beams. In the design of microsensors, optimization of geometry dimensions is essential for improving structure's performance [18-20]. Here, the width and length of supporting beams are optimized for the designed work frequency. It is seen that in Figure 1(a) all the links among the frames, anchors, and proof mass are made up of U-beams. A U-beam can be considered as the serial combination of two cantilevers, so its stiffness is simplified [21] as

$$
k_{\text {U-beam }}=\frac{E w_{\mathrm{u}}^{3} h_{\mathrm{u}}}{2 l_{\mathrm{u}}^{3}},
$$

where $E$ is the elastic modulus of silicon and $w_{\mathrm{u}}, h_{\mathrm{u}}$, and $l_{\mathrm{u}}$ are the width, the depth, and the length of the U-beam, respectively. The dual-mass gyroscope is symmetric so (3) and (4) can be simplified through $k_{1}=k_{2}$. Then, it is easily noticed that $f_{\text {ip }}$ and $f_{\text {op }}$ both increase with $k_{1}$ and $k_{2}$. Besides, their difference increases when $k_{c}$ grows larger. Therefore, the work frequency can be increased through increasing the width or decreasing the length of support beams, which is verified by the following finite element analysis.

In Figure 2, the equivalent finite element model of the gyroscope structure is developed using Ansys to analyze the influence of support beams' size on work modes' frequencies. The silicon material properties and the mesh element are listed in Table 1. During simulation, the length and width of the drive U-beams and the sense U-beams are changed separately. The simulated work frequencies, namely, the $f_{\text {op }}$ in drive and sense directions, are fitted as shown in Figure 3.

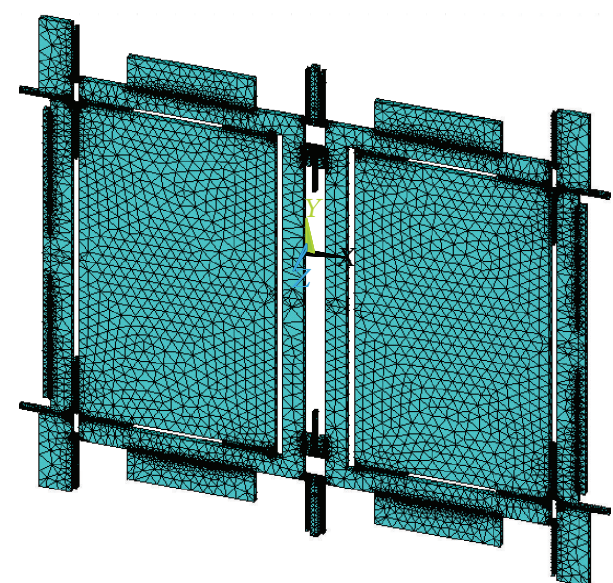

FIGURE 2: Equivalent finite element model of the dual-mass gyroscope structure.

TABLE 1: Parameters for FE simulation.

\begin{tabular}{cccc}
\hline \multicolumn{2}{c}{ Parameter } & Value & Unit \\
\hline \multirow{3}{*}{ Single-crystal silicon } & Young's modulus & $1.7 \times 10^{11}$ & $\mathrm{~Pa}$ \\
& Poisson's ratio & 0.28 & - \\
& Density & 2330 & $\mathrm{~kg} / \mathrm{m}^{3}$ \\
Mesh element & Solid 95 & - \\
\hline
\end{tabular}

In Figures 3(a) and 3(b), it is easily noticed that the frequency of sense mode varies little when the size of drive U-beams changes. Similarly, in Figures 3(c) and 3(d), the frequency of drive mode changes little when the size of the sense U-beams changes. Thus, the separate tuning effect of support beams is realized, which eases the frequency localization of the two work modes. Besides, increasing the width of the sense $\mathrm{U}$ beams or decreasing their length raises the natural frequency of sense mode. The same tuning trend occurs to the drive mode frequency when the drive U-beams change in the same way. The tuning effect, obtained by the finite element analysis, matches the theoretical expectation from (4) and (5).

3.2. Negative Electrostatic Stiffness Effect. Figure 4 is a part of the tuning combs. When the fixed plate is loaded with the voltage $V$ and the moving plate translates only in the $Y$ axis, the generated negative electrostatic stiffness along the sense direction is derived [22]:

$$
\begin{aligned}
k_{e y} & =-n \varepsilon h_{c 1} l_{c 1} V^{2}\left(\frac{1}{\left(e_{c 1}-y\right)^{3}}+\frac{1}{\left(e_{c 1}+y\right)^{3}}\right) \\
& \approx-2 \frac{n \varepsilon h_{c 1} l_{c 1}}{e_{c 1}^{3}} V^{2} \quad\left(y \ll e_{c 1}\right),
\end{aligned}
$$

where $n$ is the number of the tuning combs attached to one sense frame, $h_{c 1}, l_{c 1}$, and $e_{c 1}$ are the depth, length, and distance of the comb capacitor, and $\varepsilon$ is the absolute dielectric constant of the gap. According to (4) and Figure 3, by changing the effective system stiffness between the vibrating mass and the substrate, that is, $k_{1}$ and $k_{2}$, the work frequencies can be 


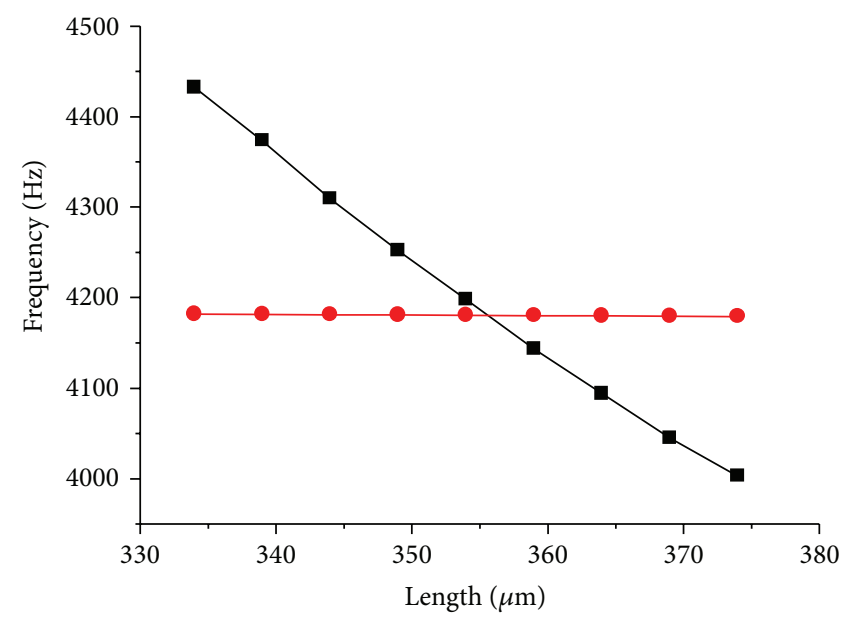

(a)

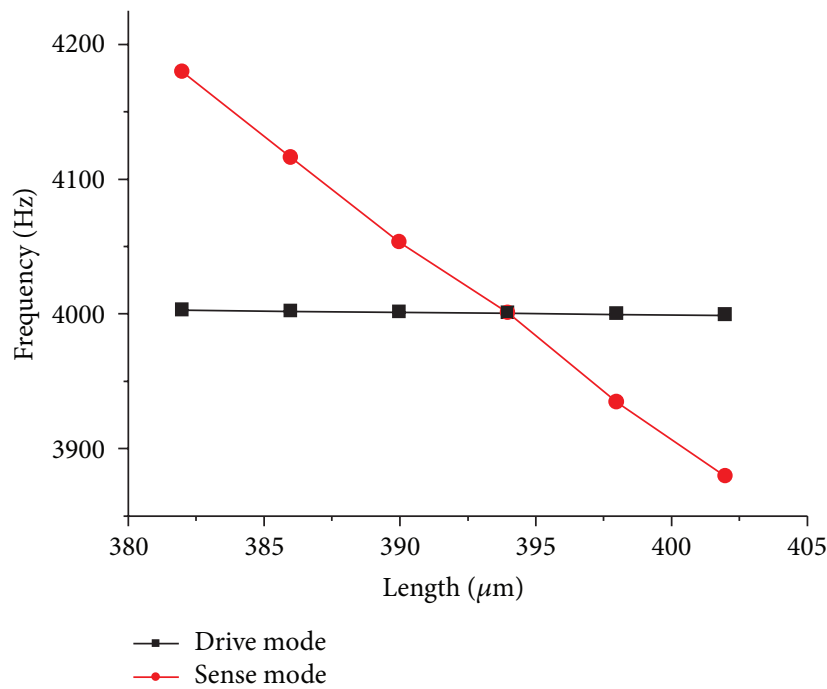

(c)

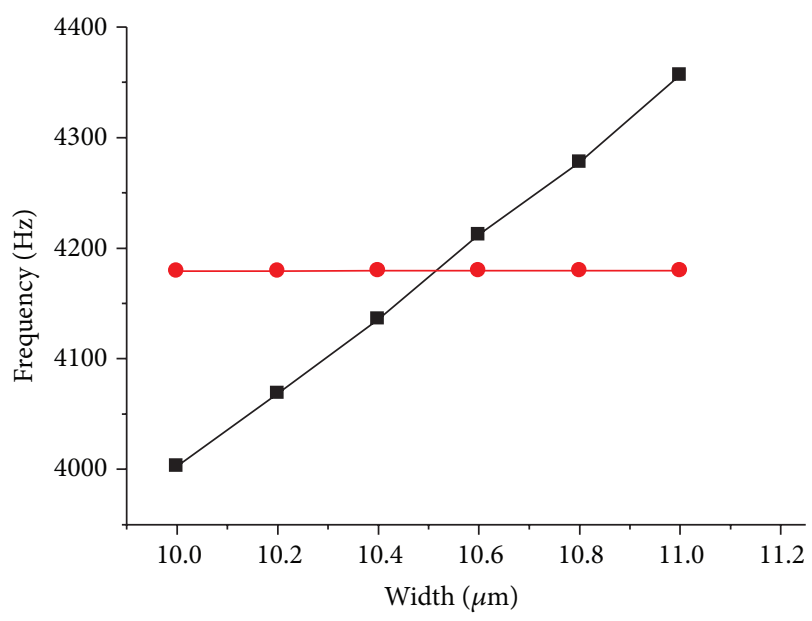

(b)

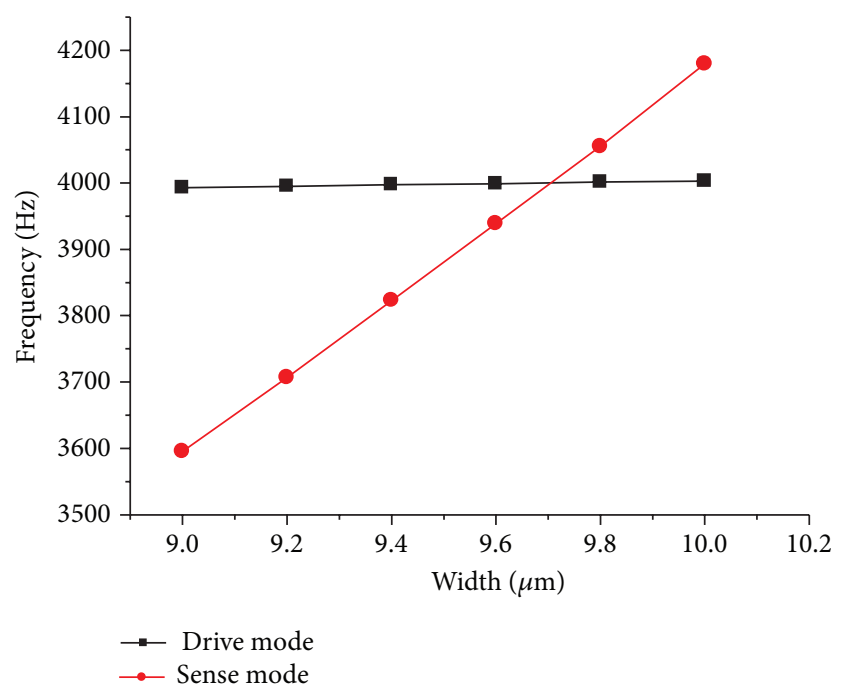

(d)

FIGURE 3: The dependence of work frequencies on the size of U-beams: (a) the length of drive U-beams, (b) the width of drive U-beams, (c) the length of sense U-beams, and (d) the width of sense U-beams.

tuned towards their expected value. Therefore, the negative electrostatic stiffness effect of the squeeze capacitors in sense frames is used to change $k_{1}$ and $k_{2}$ and modify the natural frequency of sense mode. When the tuning capacitors are loaded with $V$, the frequencies of the in-phase and antiphase translation in the sense direction are derived as seen in

$$
\begin{aligned}
f_{\text {ip } y}=\frac{1}{2 \pi} \sqrt{\frac{k_{1 y}+k_{e y}}{m_{y}}}=\frac{1}{2 \pi} \sqrt{\frac{k_{1 y}}{m_{y}}-\frac{2 n \varepsilon h_{c 1} l_{c 1}}{m_{y} e_{c 1}^{3}} V^{2}}, \\
f_{\text {op } y}=\frac{1}{2 \pi} \sqrt{\frac{k_{1 y}+2 k_{c y}+k_{e y}}{m_{y}}} \\
=\frac{1}{2 \pi} \sqrt{\frac{k_{1 y}+2 k_{c y}}{m_{y}}-\frac{2 n \varepsilon h_{c 1} l_{c 1}}{m_{y} e_{c 1}^{3}} V^{2}},
\end{aligned}
$$

where $m_{y}, k_{1 y}$, and $k_{c y}$ are the effective system mass and stiffness in the sense direction, as explained in Section 2. It is easily seen that natural frequencies of the in-phase and antiphase vibration both decrease when the tuning voltage $V$ increases.

3.3. Experiment Measurement. The dual-mass gyroscope design, with the frequency difference between work modes around $100 \mathrm{~Hz}$, is fabricated using the SOG process [23] and vacuum packaged in ceramic [24]. The frequency localization effect of the support beams and the tuning capacitors are verified by the frequency sweeping tests. As seen in Figure 5, the experimental setup is made up of the power supplies, the detect circuit board, an oscilloscope, and the packaged gyroscope. To test the frequency response of the fabricated gyroscope, $2 \mathrm{~V}$ AC biased with $5 \mathrm{~V}$ DC is applied on the drive/sense electrodes and the detect circuit is connected with one anchor to sense the vibration of the gyroscope. The 


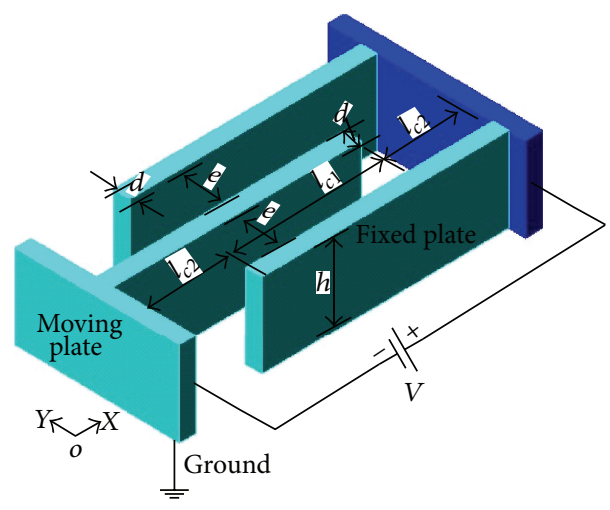

Figure 4: Part of the tuning parallel capacitors.

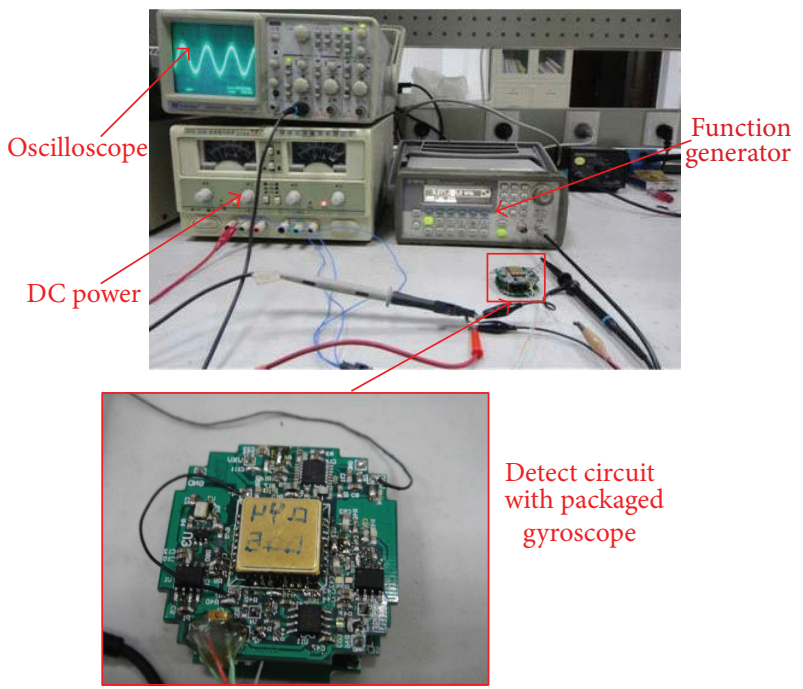

FIGURE 5: Experimental setup for frequency sweeping test.

oscilloscope is used to display the vibration signal from the circuit.

Figure 6 gives the frequency response curves of work modes, obtained by fitting the vibration amplitude (in Volt) displayed on the oscilloscope at every frequency step within the range of interest. It is known that the vibration amplitude changes along with the actuation frequency and the largest amplitude is achieved when the drive frequency is equal to the natural frequency. Thus, the work frequencies of drive mode and sense mode, $4007.9 \mathrm{~Hz}$ and $4168.1 \mathrm{~Hz}$, are obtained from the two curves. And the frequency difference after fabrication is $160.2 \mathrm{~Hz}$, much larger than the designed $100 \mathrm{~Hz}$. As the drive mode and sense mode resonators can be considered as band-pass filters, there exists a relationship between the $3 \mathrm{~dB}$ bandwidth and the quality factor, $\mathrm{BW}_{3 \mathrm{~dB}}=f_{0} / \mathrm{Q}$, where $f_{0}$ and $Q$ are the natural frequency and quality factor of each mode. Thus, the quality factor of drive mode and sense mode, around 5000 and 950, is obtained based on Figure 6 .

The frequency sweeping methods are also used to obtain the natural frequency of sense mode when varied DC voltage is applied on the tuning electrodes. Figure 7 gives the frequency tuning curves obtained by the negative electrostatic
TABLE 2: Designed structure parameters for gyroscope.

\begin{tabular}{lccc}
\hline \multicolumn{1}{c}{ Parameter } & & Value & Unit \\
\hline$X$ stiffness & $k_{1 x}$ & 302.2 & $\mathrm{~N} / \mathrm{m}$ \\
$X$ coupling stiffness & $k_{c x}$ & 74.6 & $\mathrm{~N} / \mathrm{m}$ \\
$Y$ stiffness & $k_{1 y}$ & 431.7 & $\mathrm{~N} / \mathrm{m}$ \\
$Y$ coupling stiffness & $k_{c y}$ & 20.9 & $\mathrm{~N} / \mathrm{m}$ \\
$X$ mass & $m_{x}$ & 0.68 & $\mathrm{mg}$ \\
$Y$ mass & $m_{y}$ & 0.68 & $\mathrm{mg}$ \\
& $n$ & 19 & - \\
Tuning comb & $h_{c 1}$ & 60 & $\mu \mathrm{m}$ \\
& $l_{c 1}$ & 300 & $\mu \mathrm{m}$ \\
& $e_{c 1}$ & 4.5 & $\mu \mathrm{m}$ \\
\hline
\end{tabular}

stiffness effect. It is noticed that the experimental tuning effect matches with the theoretical results, calculated by (7) using the parameter value in Table 2 . As the applied tuning voltage increases, the two natural frequencies in the sense direction ( $Y$-axis) both decrease but the two frequencies in the drive direction ( $X$-axis) are almost constant. Besides, when the tuning voltage increases, the frequency gap between the drive mode and the in-phase vibration along the sense direction (a disturbing mode) is enlarged.

With the aid of the tuning capacitors, the mode distribution of the gyroscope structure is more desirable because the frequencies of work modes are further separated from those of disturbing modes. Besides, the frequency difference between sense mode and drive mode can be adjusted from $-55.8 \mathrm{~Hz}$ to $160.2 \mathrm{~Hz}$ when the tuning voltage limit is $20 \mathrm{~V}$. Thus, the bandwidth BW can be tuned down from $86.5 \mathrm{~Hz}$ to $0 \mathrm{~Hz}$ according to different application conditions. Meanwhile, the sensitivity can be increased gradually. The experimental matching voltage $18 \mathrm{~V}$ is a bit smaller than the theoretical voltage $18.3 \mathrm{~V}$, mainly resulting from the underetching effect during fabrication.

Assuming $A_{d}=1 \mu \mathrm{m}$ and $\Omega_{\text {in }}=100 \mathrm{deg} / \mathrm{s}$, the sense amplitude of the gyroscope prototype, calculated by (1), is shown in Figure 8. It is noticed that the amplitude is increased by over 20 times when the difference is decreased from $50 \mathrm{~Hz}$ to $0 \mathrm{~Hz}$, verifying that the study about tuning frequency difference is beneficial to increase the sensitivity of the gyroscope.

\section{Automatic Tuning Algorithm}

The schematic control loop for tuning the natural frequency of sense mode towards the natural frequency of drive mode is displayed in Figure 9. When gyroscope works, the sense mode is driven by the Coriolis and quadrature coupling force. $\Omega_{\text {equ }}$ is the equivalent angular input of the quadrature coupling force. An additional external signal $A_{d t h} \cos \omega_{d t h} t$ is applied as well, where $A_{d t h}$ and $\omega_{d t h}$ are the amplitude and frequency of the signal. The response of the external signal is used as the information to modify the applied tuning voltage so as to change the natural frequency of the sense mode. 


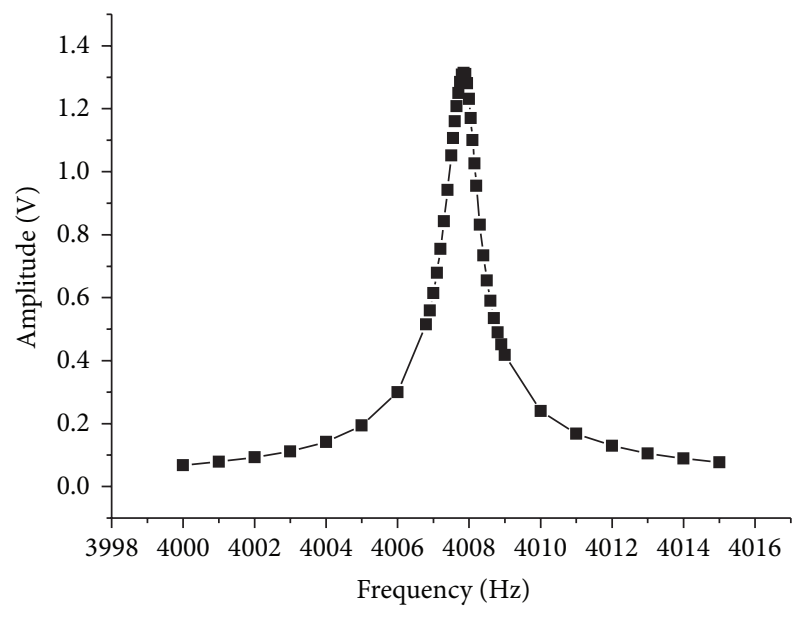

(a) Drive mode

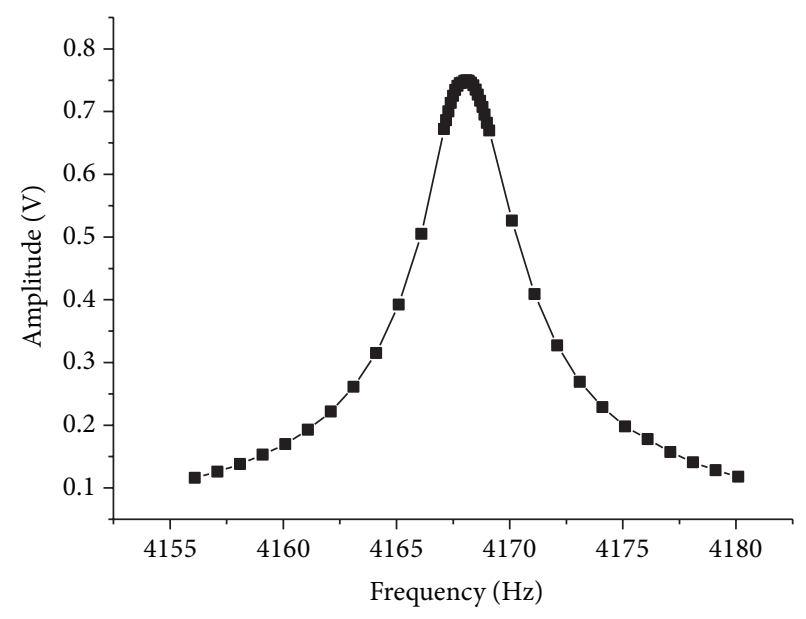

(b) Sense mode

Figure 6: The dependence of vibration amplitude on actuation frequency.

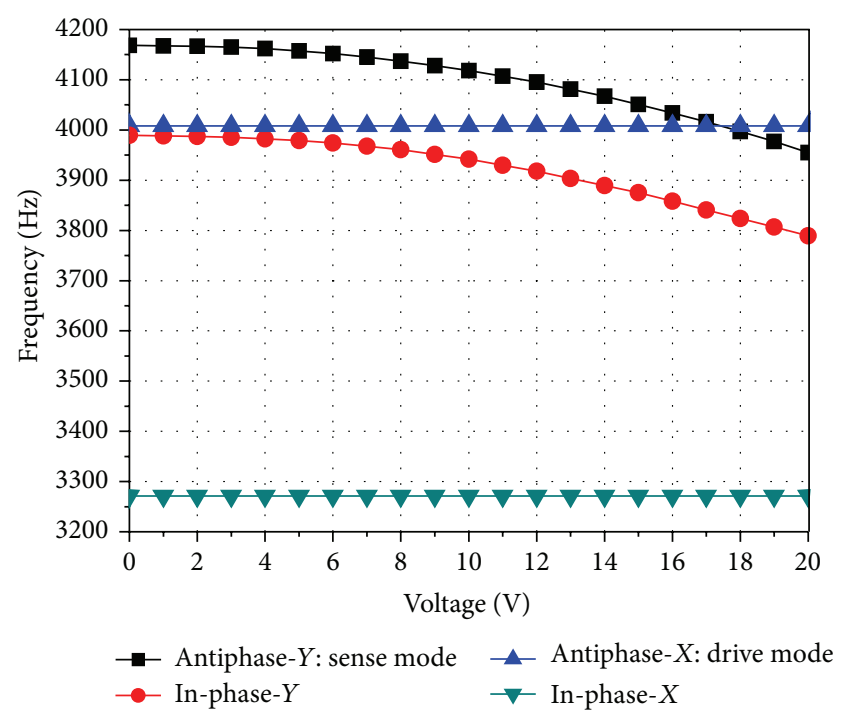

(a)

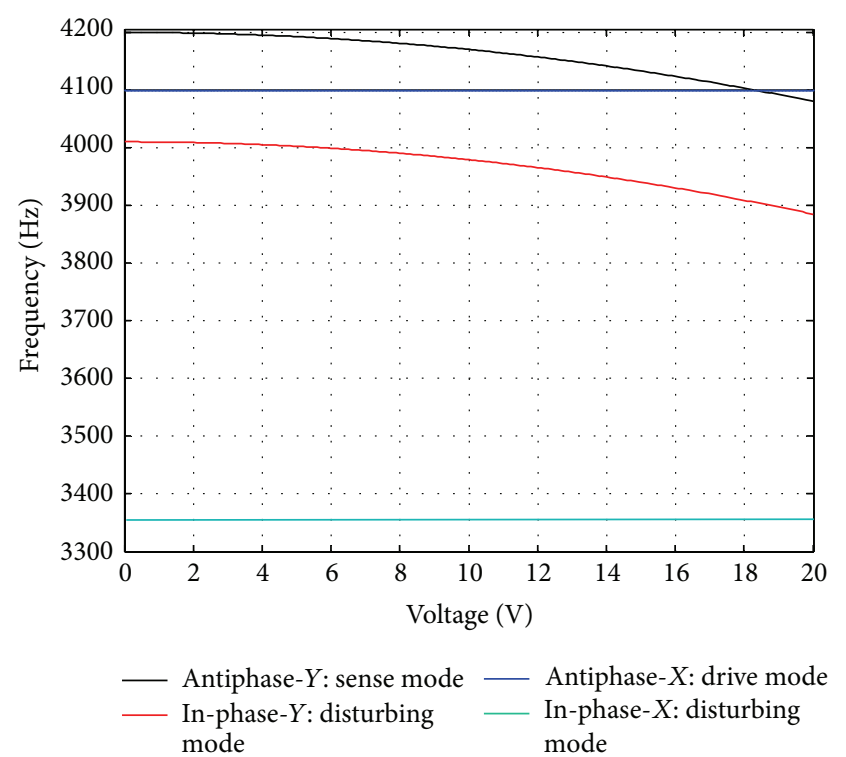

(b)

Figure 7: The dependence of the natural frequencies in drive and sense directions on the voltage applied on the tuning electrodes. (a) Experimental tuning curves. (b) Theoretical tuning curves.

The tuning algorithm is derived based on the approximate symmetry of frequency response of the second order resonator, as seen in

$$
\begin{gathered}
A_{\omega_{d}}=\frac{1 / m_{y}}{\sqrt{\left(\omega_{y}^{2}-\omega_{d}^{2}\right)^{2}+\left(\omega_{y} \omega_{d} / Q_{y}\right)^{2}}}, \\
\varphi_{\omega_{d}} \\
= \begin{cases}-\operatorname{arctg}\left(\frac{\omega_{y} \omega_{d}}{Q_{y}} \cdot \frac{1}{\omega_{y}^{2}-\omega_{d}^{2}}\right), & \omega_{d} \leq \omega_{y}, \\
-\left(180^{\circ}-\operatorname{arctg}\left(\frac{\omega_{y} \omega_{d}}{Q_{y}} \cdot \frac{1}{\omega_{d}^{2}-\omega_{y}^{2}}\right)\right), & \omega_{d}>\omega_{y},\end{cases}
\end{gathered}
$$

where $A_{\omega_{d}}$ and $\varphi_{\omega_{d}}$ are the amplitude and phase characteristic, respectively. Similarly, $A_{\omega_{d} \pm \omega_{d t h}}$ and $\varphi_{\omega_{d} \pm \omega_{d t h}}$ are the characteristic at frequency $\omega_{d} \pm \omega_{d t h}$. When the resonator is applied with two respective forces at $\omega_{d} \pm \omega_{d t h}$ and $\omega_{d}=\omega_{y}$, their responses after demodulation and filtering are nearly opposite because the two frequencies are symmetric about the natural frequency $\omega_{y}$. By comparing the two responses, the natural frequency of sense mode and the drive frequency can be in the approximate match condition. The following equations detail the algorithm and processing.

4.1. Working Principle. In the sense mode, the low frequency carrier $A_{d t h} \cos \omega_{d t h} t$ is loaded, modulated by $\sin \omega_{d} t$ with $\omega_{d t h} \ll \omega_{d}$, and then applied on the feedback combs in sense 


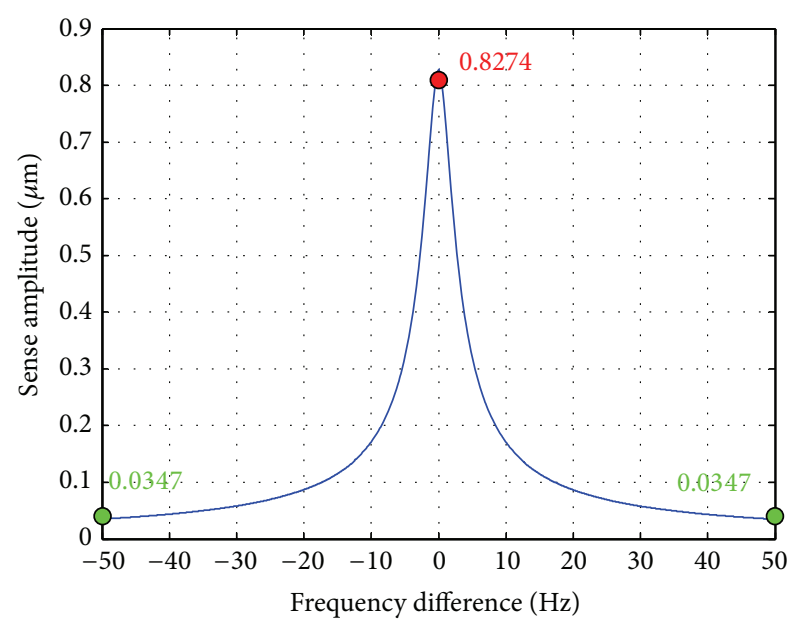

FIGURE 8: Dependence of sense amplitude on frequency difference.

frames. Then, the generated electrostatic force applied on the sense frames is

$$
\begin{aligned}
F_{d t h} & =A_{d t h} \cos \omega_{d t h} t \times \sin \omega_{d} t \times k_{d e} \\
& =\frac{1}{2} A_{d t h} \cdot k_{d e}\left[\sin \left(\omega_{d}+\omega_{d t h}\right) t+\sin \left(\omega_{d}-\omega_{d t h}\right) t\right],
\end{aligned}
$$

where $k_{d e}$ is the equivalent transfer factor of feedback combs from the applied voltage to the generated electrostatic force. Thus, the displacement of sense vibration driven by $F_{d t h}$ is

$$
\begin{aligned}
y_{d t h}= & \frac{1}{2} A_{d t h} \cdot k_{d e} \\
& \cdot\left\{A_{\omega_{d}+\omega_{d t h}} \sin \left[\left(\omega_{d}+\omega_{d t h}\right) t+\varphi_{\omega_{d}+\omega_{d t h}}\right]\right. \\
& \left.+A_{\omega_{d}-\omega_{d t h}} \sin \left[\left(\omega_{d}-\omega_{d t h}\right) t+\varphi_{\omega_{d}-\omega_{d t h}}\right]\right\} .
\end{aligned}
$$

Then, the displacement is amplified by a factor of $k_{y_{s}}$ through a capacitive interface circuit and demodulated by $-\sin \omega_{d} t$ and $\cos \omega_{d t h} t$, the process of which is expressed in

$$
\begin{aligned}
y_{d t h 2}= & \frac{1}{2} A_{d t h} \cdot k_{d e} \cdot k_{y s} \\
& \cdot\left\{A_{\omega_{d}+\omega_{d t h}} \sin \left[\left(\omega_{d}+\omega_{d t h}\right) t+\varphi_{\omega_{d}+\omega_{d t h}}\right]\right. \\
& \left.+A_{\omega_{d}-\omega_{d t h}} \sin \left[\left(\omega_{d}-\omega_{d t h}\right) t+\varphi_{\omega_{d}-\omega_{d t h}}\right]\right\} \\
& \times\left(-\sin \omega_{d} t\right) \times \cos \omega_{d t h} t \\
= & -A_{+} \cos \varphi_{+}-A_{-} \cos \varphi_{-} \\
& -A_{+} \cos \left(2 \omega_{d t h} t+\varphi_{+}\right)-A_{-} \cos \left(2 \omega_{d t h} t-\varphi_{-}\right) \\
& +A_{-} \cos \left[2\left(\omega_{d}-\omega_{d t h}\right) t+\varphi_{-}\right] \\
& +A_{+} \cos \left(2 \omega_{d} t+\varphi_{+}\right)
\end{aligned}
$$

$$
\begin{aligned}
& +A_{-} \cos \left(2 \omega_{d} t+\varphi_{-}\right) \\
& +A_{+} \cos \left[2\left(\omega_{d}+\omega_{d t h}\right) t+\varphi_{+}\right]
\end{aligned}
$$

where

$$
\begin{gathered}
A_{+/-}=\frac{A_{d t h} \cdot k_{d e} \cdot k_{y s} \cdot A_{\omega_{d}+\omega_{d t h} / \omega_{d}-\omega_{d t h}}}{8}, \\
\varphi_{+/-}=\varphi_{\omega_{d}+\omega_{d t h} / \omega_{d}-\omega_{d t h}} .
\end{gathered}
$$

Finally, the control signal, used for tuning the natural frequency of sense mode,

$$
y_{d t h 3}=-A_{+} \cos \varphi_{+}-A_{-} \cos \varphi_{-},
$$

is obtained by low-pass filtering the demodulated signal $y_{d t h 2}$. Substituting (8) into (13), the control signal is

$$
\begin{aligned}
y_{d t h 3} & =-A_{+} \cos \varphi_{+}-A_{-} \cos \varphi_{-} \\
& =\frac{A_{d t h} k_{d e} k_{y s} Q_{y}}{8 m_{y}} g(\Delta f),
\end{aligned}
$$

where

$$
\begin{aligned}
g(\Delta f) & \\
= & \left(Q_{y}\left(\left(\omega_{d}+\omega_{d t h}\right)^{2}-\left(\omega_{d}+2 \pi \Delta f\right)^{2}\right)\right) \\
& \times\left(\left(\left(\omega_{d}+2 \pi \Delta f\right)\left(\omega_{d}+\omega_{d t h}\right)\right)^{2}\right. \\
& \left.+\left(Q_{y}\left(\left(\omega_{d}+\omega_{d t h}\right)^{2}-\left(\omega_{d}+2 \pi \Delta f\right)^{2}\right)\right)^{2}\right)^{-1} \\
- & \left(Q_{y}\left(\left(\omega_{d}+2 \pi \Delta f\right)^{2}-\left(\omega_{d}-\omega_{d t h}\right)^{2}\right)\right) \\
\times & \left(\left(\left(\omega_{d}+2 \pi \Delta f\right)\left(\omega_{d}-\omega_{d t h}\right)\right)^{2}\right. \\
& \left.\quad+\left(Q_{y}\left(\left(\omega_{d}+2 \pi \Delta f\right)^{2}-\left(\omega_{d}-\omega_{d t h}\right)^{2}\right)\right)^{2}\right)^{-1}
\end{aligned}
$$

and $\Delta f$ is the frequency difference between the natural frequency $\omega_{y}$ and the drive frequency $\omega_{d}$.

Suppose $\omega_{d t h}$ is $2 \pi \times 80 \mathrm{rad} / \mathrm{s}$; the relationship between $g(\Delta f)$ and $\Delta f$ is displayed in Figure 10. As seen in (8), the amplitude characteristic as well as the phase characteristic is not strictly symmetric about the natural frequency $\omega_{y}$, leading to the equilibrium state of approximate matching. Theoretically, the stable frequency difference $\Delta f$ between drive mode and sense mode is localized at $0.8006 \mathrm{~Hz}$ by using this control loop. However, the symmetry of (8) can be enhanced when the drive frequency and the natural frequency grow larger. Thus, the difference can be tuned closer to $0 \mathrm{~Hz}$.

Based on Figure 10, the detailed control process is demonstrated when different initial frequency difference appears. It is noticed that when $-79.9738 \mathrm{~Hz}<\Delta f<0.8006 \mathrm{~Hz}$, $g(\Delta f)<0$; under the control of the closed tuning loop, the 


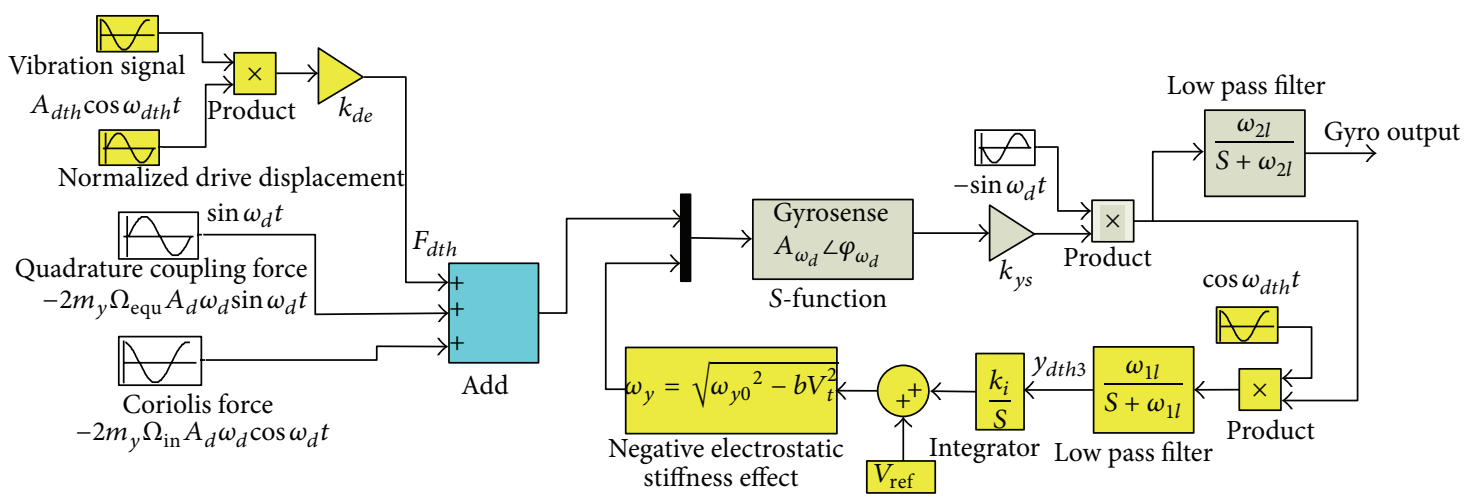

FIGURE 9: Schematic control loop for automatic frequency tuning of the sense frequency.

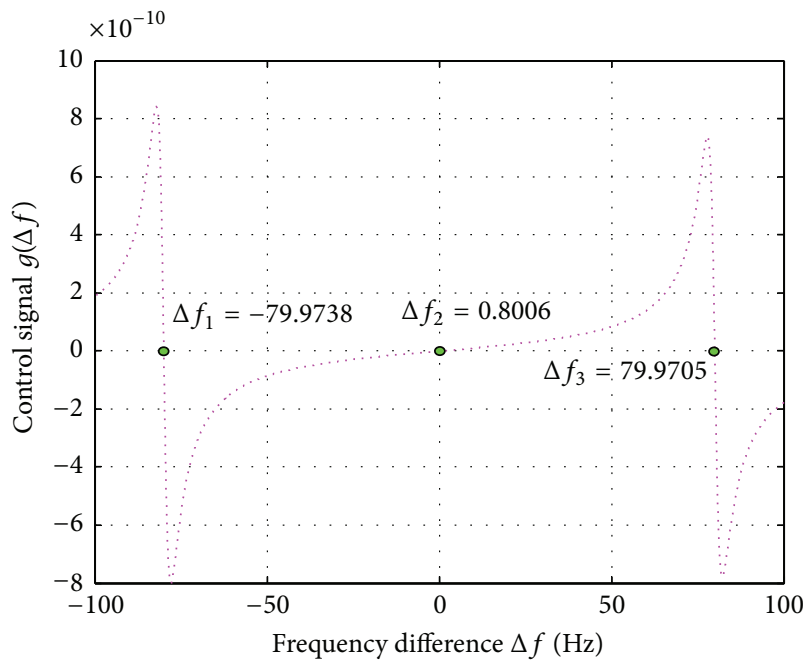

FIGURE 10: Relationship between the control signal $g(\Delta f)$ and the frequency difference $\Delta f$.

tuning voltage is decreased to enlarge $\omega_{y}$ to approach $\omega_{d}$. Similarly, when $0.8006 \mathrm{~Hz}<\Delta f<79.9705 \mathrm{~Hz}, g(\Delta f)>0$ increases the tuning voltage to make $\omega_{y}$ smaller and approach $\omega_{d}$. However, when $\Delta f<-79.9738 \mathrm{~Hz}, g(\Delta f)>0$; if the tuning voltage is not limited, the natural frequency is further decreased and $\Delta f$ is away from $-79.9738 \mathrm{~Hz}$ towards $-\infty$. Similarly, when $\Delta f>79.9705 \mathrm{~Hz}, g(\Delta f)<0$; the natural frequency is increased until it reaches its initial value and $\Delta f$ is larger than $79.9705 \mathrm{~Hz}$. Thus, the initial frequency difference should be controlled within $[-79.9738 \mathrm{~Hz}, 79.9705 \mathrm{~Hz}]$ so that the stable difference can converge to $0.8006 \mathrm{~Hz}$.

\subsection{Simulation Verification}

4.2.1. Frequency Response. The simulation model in Figure 9 is built using Matlab and Simulink. The sense mode vibration is developed using the $S$-Function module with two inputs; one is the total applied force and the other is the tuned sense frequency by the negative electrostatic stiffness effect. The simulation parameters are shown in Table 3 , in which $b$ is approximated according to Figure 7(a). As the initial
TABLE 3: Simulation parameters for frequency control loop.

\begin{tabular}{lc}
\hline Parameters & Value \\
\hline$k_{d e}$ & $1 \times 10^{-6} \mathrm{~N} / \mathrm{V}$ \\
$k_{y s}$ & $1 \times 10^{10} \mathrm{~V} / \mathrm{m}$ \\
$v_{\text {ref }}$ & $17 \mathrm{~V}$ \\
$A_{d t h}$ & $0.001 \mathrm{~V}$ \\
$\omega_{d t h}$ & $2 \pi \times 80 \mathrm{rad} / \mathrm{s}$ \\
$\omega_{1 l}$ & $2 \pi \times 10 \mathrm{rad} / \mathrm{s}$ \\
$\omega_{2 l}$ & $2 \pi \times 10 \mathrm{rad} / \mathrm{s}$ \\
$m_{y}$ & $0.68 \times 10^{-6} \mathrm{~kg}$ \\
$\Omega_{\text {in }}$ & $100 \mathrm{deg} / \mathrm{s}$ \\
$\Omega_{\text {equ }}$ & $100 \mathrm{deg} / \mathrm{s}$ \\
$b$ & 157580 \\
\hline
\end{tabular}

difference of $160.2 \mathrm{~Hz}$ between drive mode and sense mode is out of the range of the desirable difference shown in Figure 10, a tuning voltage $V_{\text {ref }}$ is preloaded on the tuning combs to make the frequency difference below $79 \mathrm{~Hz}$. An integrator is used as the controller to tune the voltage applied on the tuning combs.

When the gyroscope works with the Coriolis and quadrature coupling force applied, the response of the frequency control loop, namely, the tuning voltage and the sense frequency, is given in Figure 11. Besides, the integrating parameter $k_{i}$ is optimized for fast response and small overshoot. It is noticed that when $k_{i}$ increases from 10 to 60 , the equilibrium time decreases but the oscillation at the beginning grows to be severe. When $k_{i}=30$, the control loop can be stabilized within 8 seconds with the final tuning voltage around $18.01 \mathrm{~V}$.

The natural frequency response under different force loads is shown in Figure 12. When no Coriolis force or quadrature force is applied, the stable frequency difference is around $0.8 \mathrm{~Hz}$, matching the theoretical difference. But when a large force is applied on the sense mode, the frequency control signal $y_{d t h 3}$ will be contaminated, causing different stable difference. It is seen that when only the Coriolis force is applied and $\Omega_{\text {in }}=100 \mathrm{deg} / \mathrm{s}$, the difference turns to $0.3 \mathrm{~Hz}$. If the quadrature force, whose equivalent angular signal $\Omega_{\text {equ }}=$ $100 \mathrm{deg} / \mathrm{s}$, is applied as well, the difference is around $1.6 \mathrm{~Hz}$. It is concluded that the difference distributes around $0.8 \mathrm{~Hz}$ 


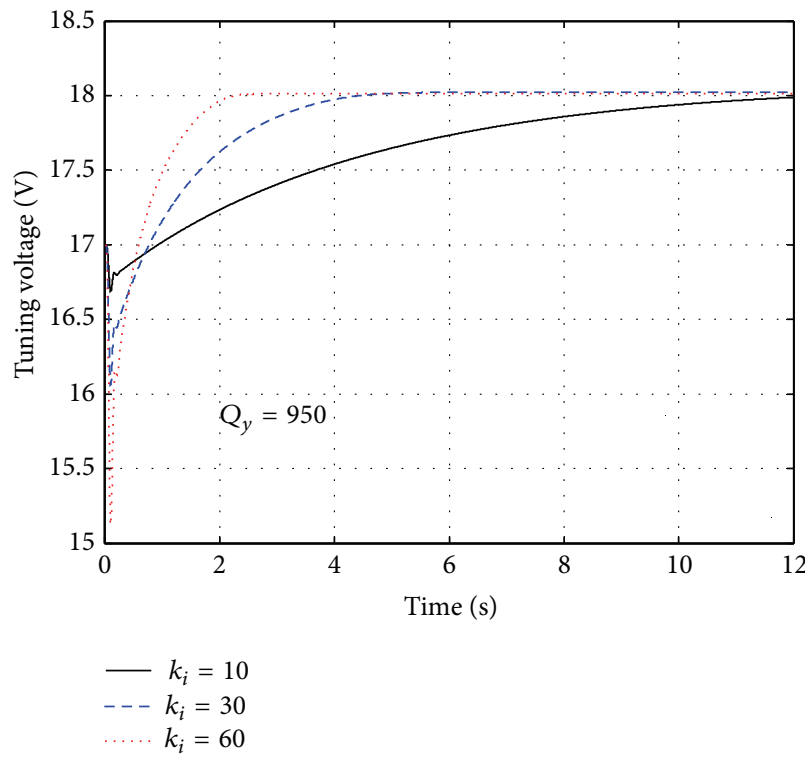

(a) Tuning voltage

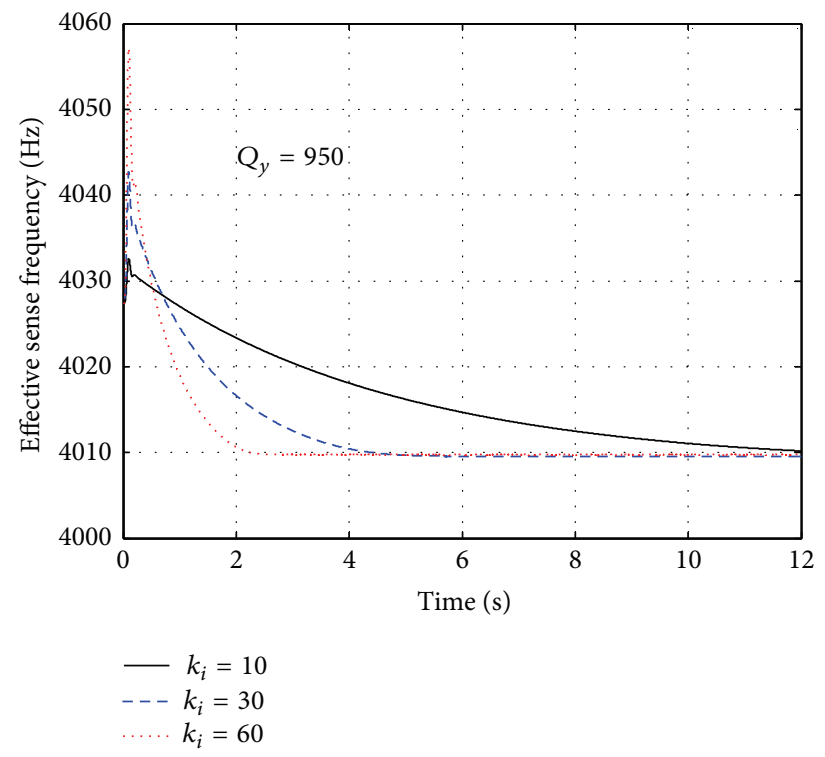

(b) Sense frequency

FIGURE 11: Response of the tuning voltage $V$ and the sense frequency $f_{y}$ when $k_{i}$ varies.

when the gyroscope works and it approaches $0.8 \mathrm{~Hz}$ when the applied force decreases. Thus, when the applied force is large, a force feedback loop is needed to diminish the influence of the Coriolis and quadrature force on the frequency difference.

Besides, the influence of the quality factor $Q_{y}$ is investigated, as displayed in Figure 13. It is shown that the frequency response is almost the same when $Q_{y}$ varies from 200 to 2000 , indicating that the frequency control loop is independent on $Q_{y}$ within the simulated range. However, as $Q_{y}$ grows larger, the stable sense frequency vibrates in sine wave, caused by the enlarged gyroscope response under the Coriolis and quadrature force.

4.2.2. Gyro Output. In Figure 9, two forces are applied; one is the useful signal, the Coriolis force, and the other is the disturbing signal, the quadrature coupling force. As seen in the control loop, the gyro output is obtained by demodulating the gyroscope response. If the drive frequency is not equal to the natural frequency, namely, the frequency difference is not $0 \mathrm{~Hz}$, the phase delay of the sense resonator is not 90 degree. Then, during the demodulation by $-\sin \omega_{d} t$, the gyro output includes the element influenced by the quadrature signal. Therefore, as seen in Figure 14, the gyro output, obtained by simulating the system in Figure 9, is different when the quadrature coupling is present and absent. The useful gyro output signal is contaminated by the quadrature coupling. Thus, the quadrature coupling should be compensated to decrease the output error when this frequency tuning loop is used. The quadrature compensation is discussed in $[25,26]$, and in the following simulation the quadrature coupling is assumed to be 0 .

The response of the gyro output under different input angular rate, $\Omega_{\text {in }}=A_{\text {win }} \cos \left(2 \pi f_{\text {win }} t\right)$, is illustrated in Figure 15. As shown in Figure 12, the frequency tuning loop

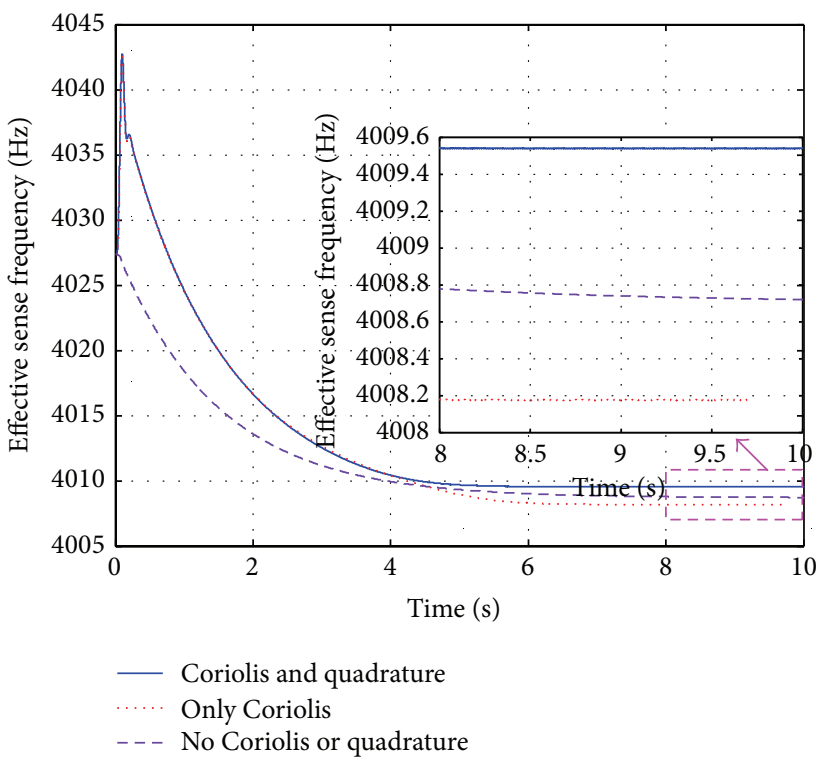

FIGURE 12: Sense frequency under different applied force.

takes nearly 8 seconds to make the natural frequency of sense mode reach its equilibrium state. Besides, it is known that the gyroscope output is dependent on the difference between the natural frequency and the drive frequency. Thus, as displayed in Figure 15(a), during the initial 8 seconds the gyroscope output gradually increases as the natural frequency of sense mode approaches the drive frequency. When $f_{\text {win }} \neq 0$, the maximum output happens when the corresponding drive frequency $f_{d} \pm f_{\text {win }}$ equals the natural frequency. Therefore, when $f_{\text {win }}$ is $5 \mathrm{~Hz}$, the maximum output happens at $4012.9 \mathrm{~Hz}$, which explains why a peak appears 
TABLE 4: Frequency tuning rate for drive mode and sense mode.

\begin{tabular}{lccccc}
\hline & \multicolumn{2}{c}{$\begin{array}{c}\text { Tuning rate of drive U-beams }(\mathrm{Hz} / \mu \mathrm{m}) \\
\text { width }\end{array}$} & length & $\begin{array}{c}\text { Tuning rate of sense U-beams }(\mathrm{Hz} / \mu \mathrm{m}) \\
\text { width }\end{array}$ & Tuning rate of capacitors $(\mathrm{Hz} / \mathrm{V})$ \\
\hline Drive mode & 353.5 & -10.7 & 0 & 0 & 0 \\
Sense mode & 0 & 0 & 583.7 & -15.0 & -10.8 \\
\hline
\end{tabular}

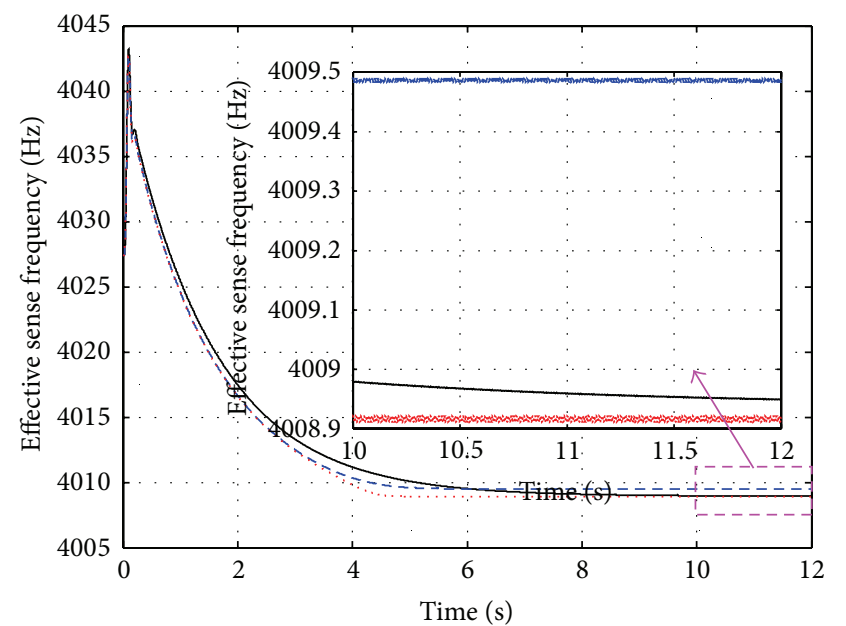

$$
\begin{aligned}
& -Q=200 \\
& ---Q=1000 \\
& \cdots Q \quad Q=2000
\end{aligned}
$$

FIGURE 13: Response of the sense frequency $f_{y}$ when $Q_{y}$ varies.

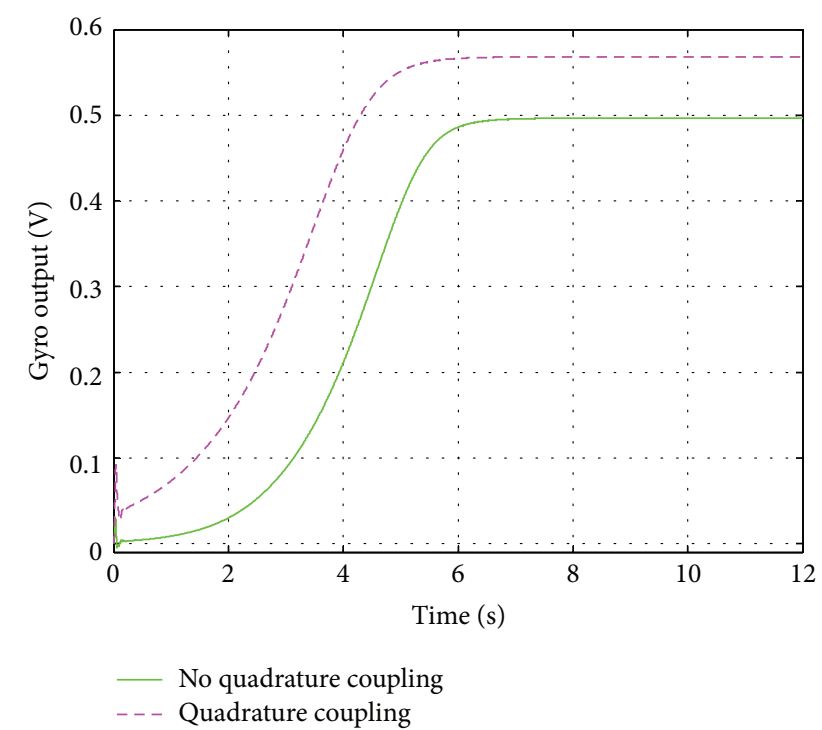

FIGURE 14: Responses of gyro output when the quadrature force is present and absent.

at the initial transient stage in Figure 15(b). In Figure 15(c), in the first 8 seconds, $f_{\text {win }}$ is maintained at $0 \mathrm{~Hz}$; after the output reaches its stable value, $f_{\text {win }}$ increases along with time in a slope of $0.1 \mathrm{~Hz} / \mathrm{s}$, that is, $f_{\text {win }}=0.1(t-8)$, and the output decreases accordingly, matching the phenomenon in
Figure 15(b). Finally, in Figure 15(c) the $3 \mathrm{~dB}$ bandwidth is calculated to be $0.836 \mathrm{~Hz}$. It is noticed that when the input angular range reaches $200 \mathrm{deg} / \mathrm{s}$, the frequency control loop is working properly. Besides, the loop is still suitable for the dynamic angular input.

\section{Results and Discussion}

The frequency tuning method of the dual-mass gyroscope is investigated in this paper from two aspects; one is to modify the size of the drive and sense U-beams, respectively, before fabrication; the other is to change the voltage applied on the tuning capacitors after fabrication. The frequency tuning rate of the support beams and the tuning capacitors are summarized in Table 4 . Width/length tuning rate is the frequency change when the width/length of support beams in drive or sense mode varies by $1 \mu \mathrm{m}$. Similarly, the capacitor tuning rate is the frequency change when the applied tuning voltage varies by $1 \mathrm{~V}$. It is indicated that the width-tuning rate is much larger than the length-tuning rate, but the variation range of the width is smaller by two orders of magnitude than that of the length in the gyroscope structure. In general, frequency localization of the work modes is implemented independently for the $Z$-axis dual-mass silicon microgyroscope by tuning the size of the support beams or the voltage applied on the tuning capacitors.

The frequency difference between work modes is set at $100 \mathrm{~Hz}$ in the structure design procedure but the tested difference of the gyroscope sample is $160.2 \mathrm{~Hz}$. By using the negative electrostatic stiffness of the squeeze capacitors in the sense frames, the difference can be tuned from $160.2 \mathrm{~Hz}$ down to $-55.8 \mathrm{~Hz}$ when the tuning voltage limit is $20 \mathrm{~V}$. Thus, the bandwidth BW can be tuned down from $86.5 \mathrm{~Hz}$ to $0 \mathrm{~Hz}$. Besides, the sensitivity can be increased by over 20 times when the difference is decreased from $50 \mathrm{~Hz}$ to $0 \mathrm{~Hz}$.

Furthermore, according to the frequency characteristic of the second order resonator, the automatic frequency control loop is successfully designed to match the frequencies of the drive and sense modes. According to the theoretical and simulation analysis, the frequency difference can be stabilized at $0.8 \mathrm{~Hz}$ when no Coriolis force or quadrature force is applied, as seen in Figures 10 and 12. When a large force is applied on the sense mode, the difference is varied; when $\Omega_{\text {in }}=100 \mathrm{deg} / \mathrm{s}$ and $\Omega_{\text {equ }}=100 \mathrm{deg} / \mathrm{s}$, the difference is $1.6 \mathrm{~Hz}$, as displayed in Figure 12; it is concluded that an additional force feedback loop is needed to suppress the disturbance when a large force is applied. Besides, quadrature compensation is needed as the gyro output is influenced by the quadrature signal, caused by the nonzero frequency difference. It is also proved that the frequency control loop 


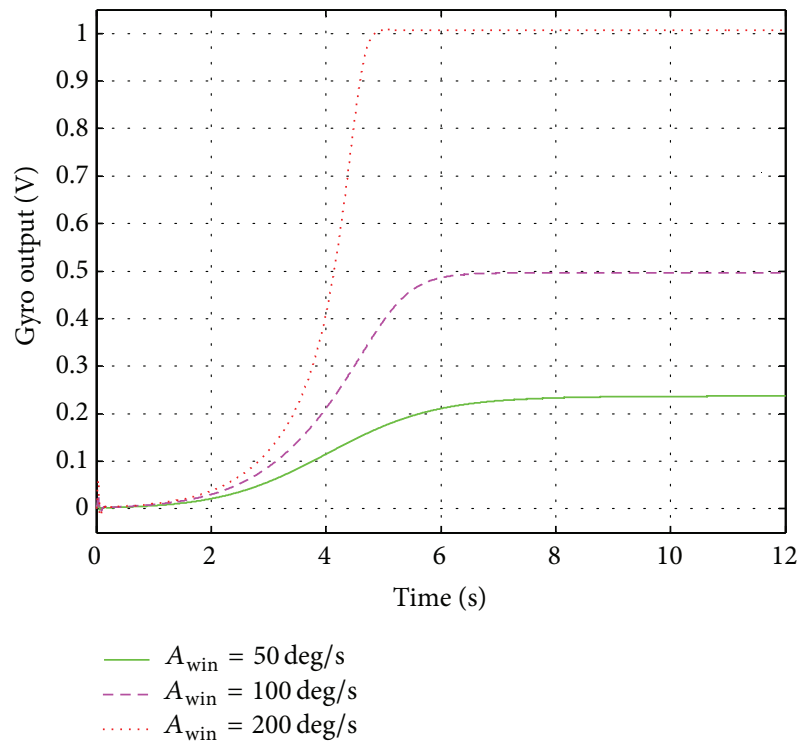

(a) $f_{\text {win }}=0$ and $A_{\text {win }}$ varies

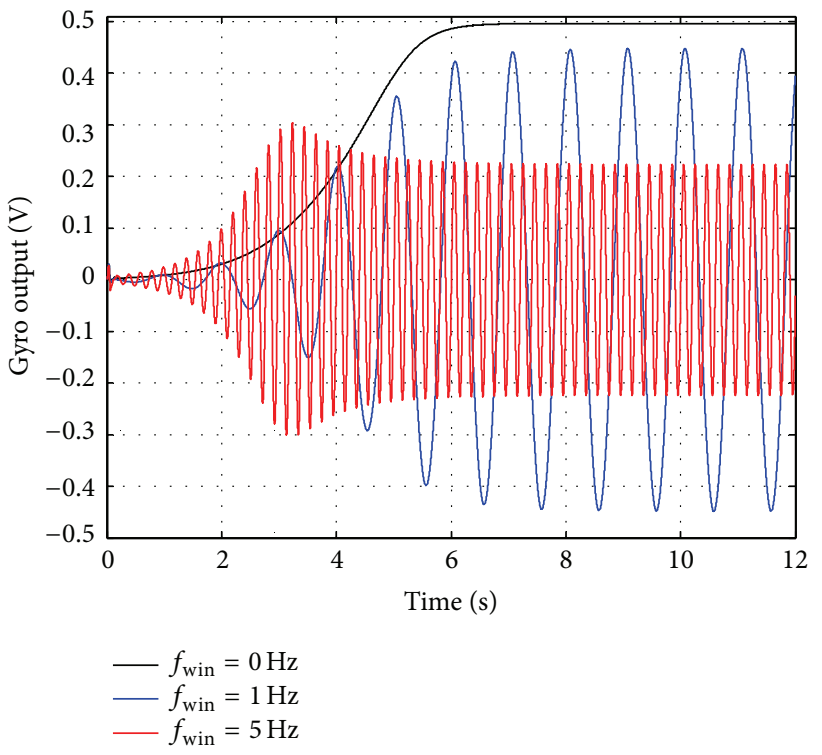

(b) $A_{\text {win }}=100 \mathrm{deg} / \mathrm{s}$ and $f_{\text {win }}$ varies

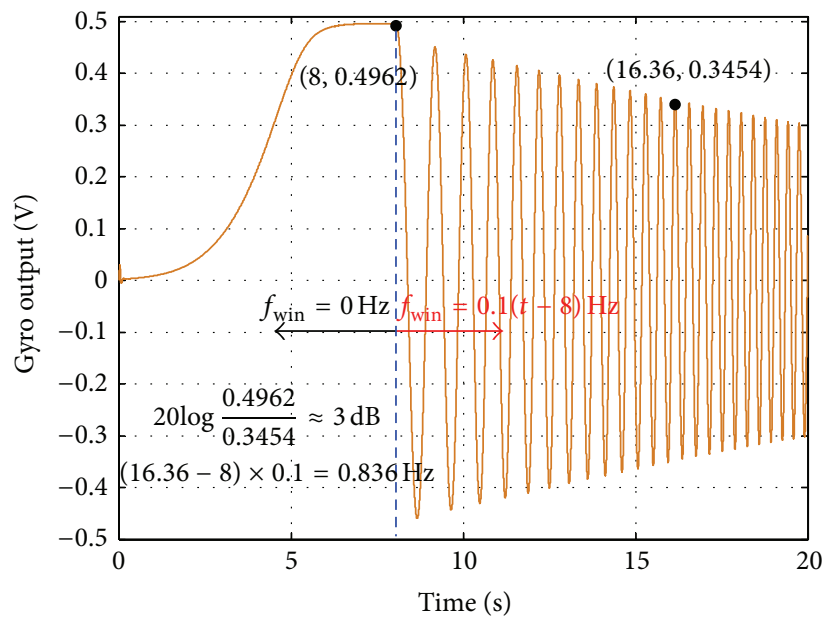

(c) $A_{\text {win }}=100 \mathrm{deg} / \mathrm{s}$ and $f_{\text {win }}$ increases along with time in a slope of $0.1 \mathrm{~Hz} / \mathrm{s}$

FIGURE 15: Responses of gyro output when the input angular rate $\Omega_{\text {in }}=A_{\text {win }} \cos \left(2 \pi f_{\text {win }} t\right)$ is applied.

can work properly under varied angular input, as shown in Figure 15. The dynamic $3 \mathrm{~dB}$ bandwidth, $0.836 \mathrm{~Hz}$, is calculated when $f_{\text {win }}$ varies along with time in a slope of $0.1 \mathrm{~Hz} / \mathrm{s}$.

Therefore, the electrostatic frequency tuning method is able to modify the frequency difference after fabrication, which eases the frequency accuracy required in the fabrication process. Besides, the automatic frequency control loop is able to tune the natural frequency towards the drive frequency when the gyroscope works with the Coriolis and quadrature force applied. When the applied force is small, the difference is almost not influenced by the force. On the other hand, when the force is large, a force feedback is required to suppress the disturbance so as to keep the difference unvaried when $s$ dynamic input is applied.

\section{Conclusion}

This paper systematically demonstrates the frequency localization of a dual-mass gyroscope from two aspects; one is to optimize the support beams' size and the other is to take advantage of the negative electrostatic stiffness effect of the squeeze capacitors. As the fabrication imperfection influences the frequency difference between the work modes randomly, the experiment results show that the tested difference is $160.2 \mathrm{~Hz}$ much larger than the designed $100 \mathrm{~Hz}$. However, the frequency difference can be localized between $-55.8 \mathrm{~Hz}$ and $160.2 \mathrm{~Hz}$ by manually applying a proper voltage under $20 \mathrm{~V}$ on the tuning capacitors. Besides, the automatic frequency control loop is designed to tune the natural frequency of sense mode towards the natural frequency of drive 
mode. This method makes the frequency difference resistive to the environment disturbances, such as the variation of the ambient temperature and work frequencies. The simulated results indicate the difference can be stabilized at $0.8 \mathrm{~Hz}$ within 8 seconds when no Coriolis force or quadrature coupling force is applied. Besides, the control loop still works when the input angular rate varies dynamically; $\Omega_{\text {in }}=$ $A_{\text {win }} \cos \left(2 \pi f_{\text {win }} t\right)$. Therefore, the study made in this paper ensures that the frequency difference can be controlled for performance improvement as well as disturbance robustness in silicon vibratory microgyroscopes.

\section{Conflict of Interests}

The authors declare that there is no conflict of interests regarding the publication of this paper.

\section{Acknowledgments}

This work is supported by the Preresearch Fund Project of 9140A09011313JW06119 and the Jiangsu Province ScienceTechnology Support Plan Project of BE2014003-3.

\section{References}

[1] A. M. Shkel, "Micromachined gyroscopes: challenges, design solutions, and opportunities," in Smart Structures and Materials 2001: Smart Electronics and MEMS, vol. 4334 of Proceedings of SPIE, pp. 74-85, Newport Beach, Calif, USA, 2001.

[2] N. Yazdi, F. Ayazi, and K. Najafi, "Micromachined inertial sensors," Proceedings of the IEEE, vol. 86, no. 8, pp. 1640-1658, 1998.

[3] J. Bernstein, S. Cho, A. T. King, A. Kourepenis, P. Maciel, and M. Weinberg, "A micromachined comb-drive tuning fork rate gyroscope," in Proceedings of the IEEE Micro Electro Mechanical Systems (MEMS '93), pp. 143-148, Fort Lauderdale, Fla, USA, February 1993.

[4] M. S. Weinberg and A. Kourepenis, "Error sources in in-plane silicon tuning-fork MEMS gyroscopes," Journal of Microelectromechanical Systems, vol. 15, no. 3, pp. 479-491, 2006.

[5] A. A. Trusov, A. R. Schofield, and A. M. Shkel, "Micromachined rate gyroscope architecture with ultra-high quality factor and improved mode ordering," Sensors and Actuators A: Physical, vol. 165, no. 1, pp. 26-34, 2011.

[6] Y. Yin, S. Wang, C. Wang, and P. Sheng, "Structural scheme design and simulation of structure-decoupled dual-mass gyroscope," Journal of Southeast University (Natural Science Edition), vol. 38, pp. 918-922, 2008.

[7] M. F. Zaman, A. Sharma, Z. Hao, and F. Ayazi, "A modematched silicon-yaw tuning-fork gyroscope with subdegreeper-hour Allan deviation bias instability," Journal of Microelectromechanical Systems, vol. 17, no. 6, pp. 1526-1536, 2008.

[8] M. F. Zaman, A. Sharma, and F. Ayazi, "High performance matched-mode tuning fork gyroscope," in Proceedings of the 19th IEEE International Conference on Micro Electro Mechanical Systems (MEMS '06), pp. 66-69, Istanbul, Turkey, 2006.

[9] D. Joachim and L. Lin, "Characterization of selective polysilicon deposition for MEMS resonator tuning," Journal of Microelectromechanical Systems, vol. 12, no. 2, pp. 193-200, 2003.
[10] T. Remtema and L. Lin, "Active frequency tuning for micro resonators by localized thermal stressing effects," Sensors and Actuators A: Physical, vol. 91, no. 3, pp. 326-332, 2001.

[11] W. Sung, M. Dalal, and F. Ayazi, "A mode-matched $0.9 \mathrm{MHz}$ single proof-mass dual-axis gyroscope," in Proceedings of the 16th International Solid-State Sensors, Actuators and Microsystems Conference (TRANSDUCERS '11), pp. 2821-2824, 2011.

[12] S. Sung, W.-T. Sung, C. Kim, S. Yun, and Y. J. Lee, "On the mode-matched control of MEMS vibratory gyroscope via phase-domain analysis and design," IEEE/ASME Transactions on Mechatronics, vol. 14, no. 4, pp. 446-455, 2009.

[13] R. Antonello, R. Oboe, L. Prandi, and F. Biganzoli, "Automatic mode matching in MEMS vibrating gyroscopes using extremum-seeking control," IEEE Transactions on Industrial Electronics, vol. 56, no. 10, pp. 3880-3891, 2009.

[14] M. Braxmaier, A. Gaißer, T. Link et al., "Cross-coupling of the oscillation modes of vibratory gyroscopes," in Proceedings of the 12th International Conference on TRANSDUCERS, Solid-State Sensors, Actuators and Microsystems, pp. 167-170, 2003.

[15] S. E. Alper and T. Akin, "A single-crystal silicon symmetrical and decoupled gyroscope on insulating substrate," in Proceedings of the 12th International Conference on TRANSDUCERS, Solid-State Sensors, Actuators and Microsystems, vol. 2, pp. 13991402, 2003.

[16] L. Huang, Y. Ni, and Y. Yin, "Fabrication error analysis on dualmass silicon micro-gyroscope," in Proceedings of the International Conference on Electrical and Control Engineering (ICECE '11), pp. 3931-3934, Yichang, China, September 2011.

[17] B. Goodwine, Engineering Differential Equations, Springer, 2010.

[18] M. Bagherinia, M. Bruggi, A. Corigliano, S. Mariani, and E. Lasalandra, "Geometry optimization of a Lorentz force, resonating MEMS magnetometer," Microelectronics Reliability, vol. 54, no. 6-7, pp. 1192-1199, 2014.

[19] K. Abbas, S. Alaie, and Z. C. Leseman, "Design and characterization of a low temperature gradient and large displacement thermal actuators for in situ mechanical testing of nanoscale materials," Journal of Micromechanics and Microengineering, vol. 22, no. 12, Article ID 125027, 2012.

[20] X. Qian and O. Sigmund, "Topological design of electromechanical actuators with robustness toward over- and underetching," Computer Methods in Applied Mechanics and Engineering, vol. 253, pp. 237-251, 2013.

[21] M. Liu, Study on the structural design technology of the symmetric and decoupling silicon micro-mechanical gyroscope [Ph.D. dissertation], School of Instrument Science and Engineering, School of Instrument Science and Engineering, Southeast University, Nanjing, China, 2007.

[22] W. A. Clark, R. T. Howe, and T. Juneau, "Micromachined vibratory rate gyroscope," Google Patents, 2000.

[23] X. He and Y. Su, "Fabrication of Z-axis micromachined gyroscope with DDSOG process," Journal of Southeast University (Natural Science), vol. 35, no. 4, pp. 545-548, 2005.

[24] Q. Shi, R. Ding, Y. Su, and A. Qiu, "Device-level vacuum packaging of silicon microgyroscopes," Journal of Mechanical Engineering, vol. 45, no. 2, pp. 243-246, 2009.

[25] B. Yang, B. Zhou, S. Wang, L. Huang, and Y. Yin, "A quadrature error and offset error suppression circuitry for Silicon MicroGyroscope," in Proceedings of the 3rd IEEE International Conference on Nano/Micro Engineered and Molecular Systems (NEMS '08), pp. 422-426, January 2008. 
[26] Y. Ni, H. Li, L. Huang, and L. Zhao, "Design and test of quadrature correction structure for silicon micro-gyroscope," Journal of Southeast University (Natural Science), vol. 43, no. 6, pp. 1227-1231, 2013. 

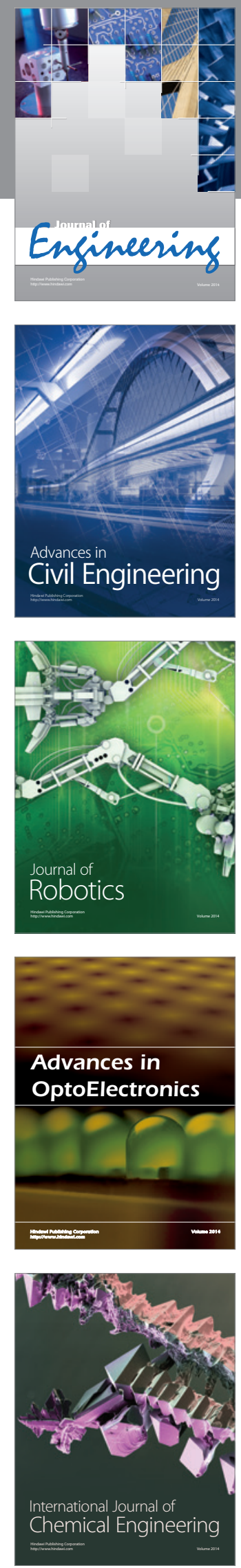

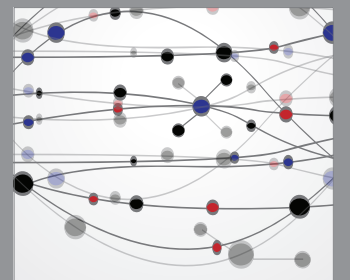

The Scientific World Journal
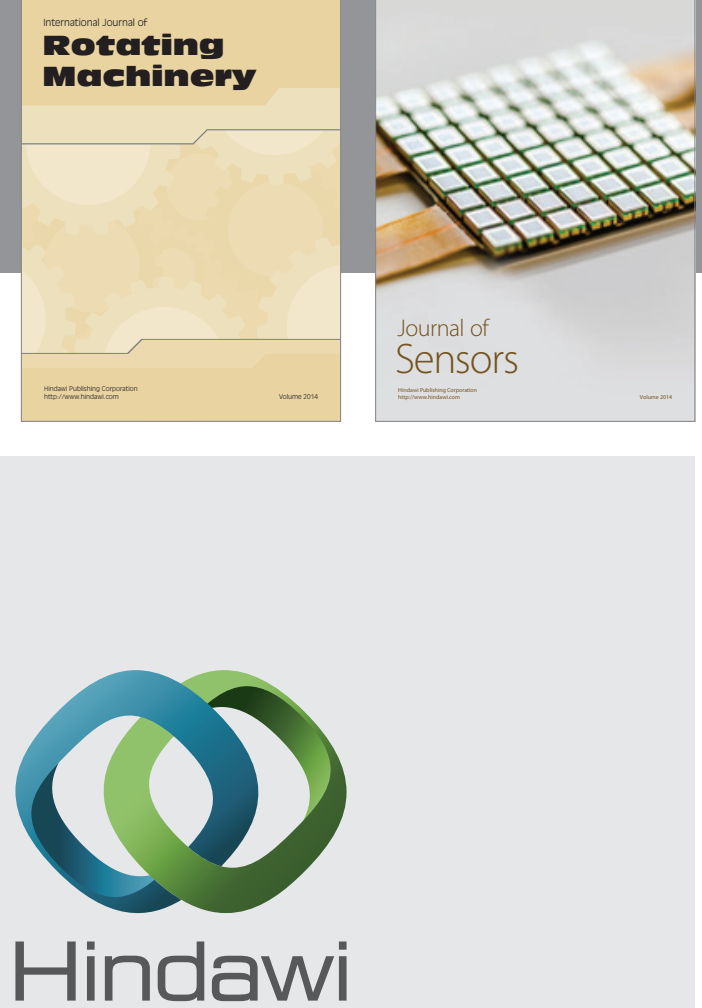

Submit your manuscripts at http://www.hindawi.com
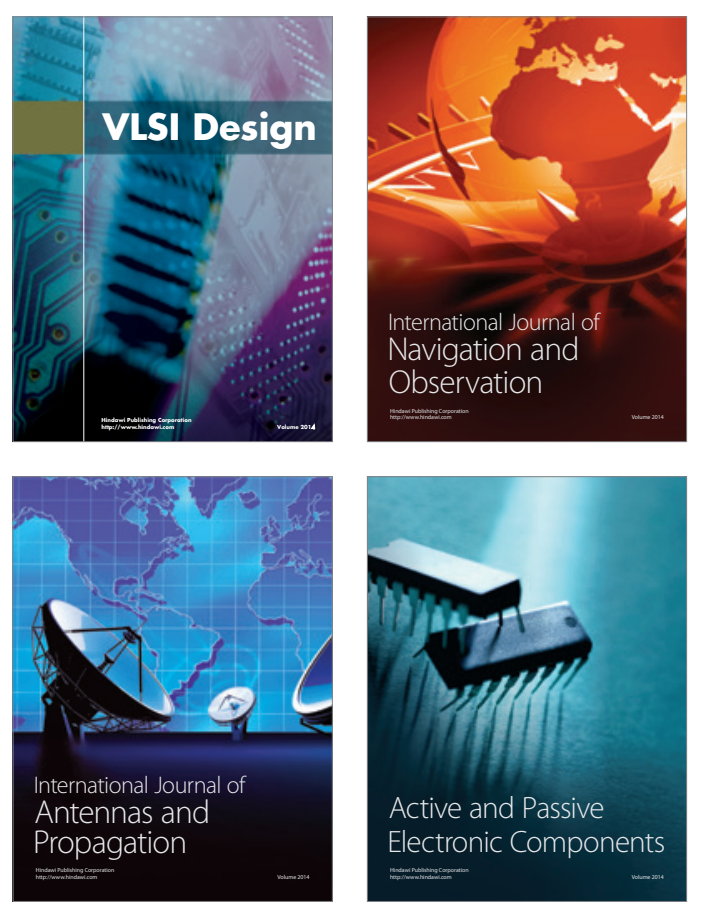
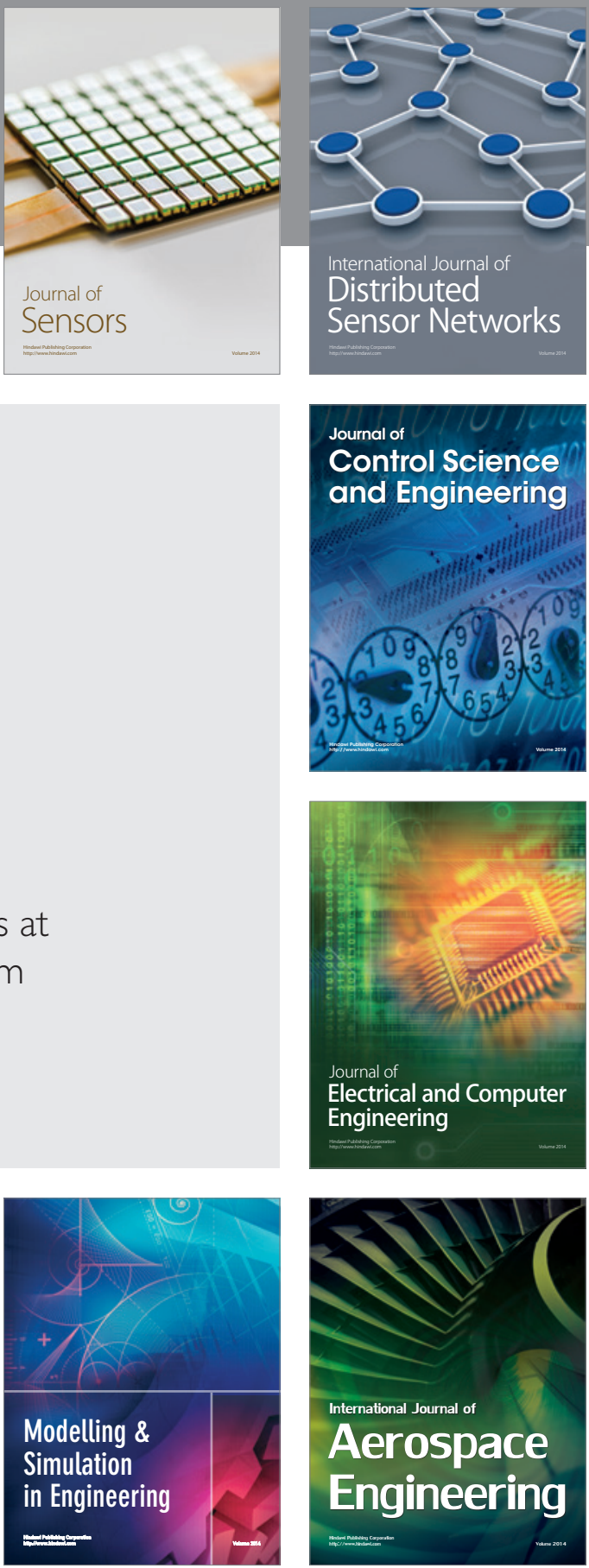

Journal of

Control Science

and Engineering
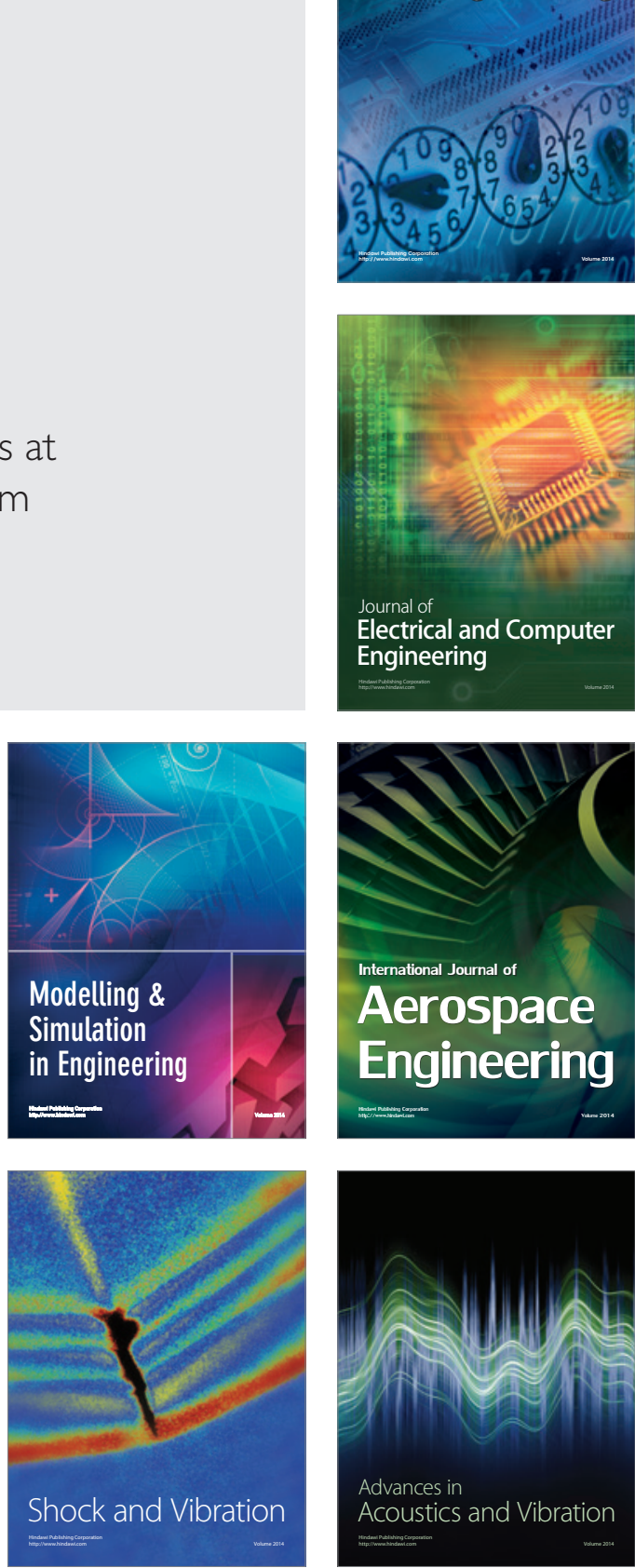